\title{
CORPUS ORAL PARA EL ESTUDIO DE LA ADQUISICIÓN Y APRENDIZAJE DEL COMPONENTE FÓNICO DEL ESPAÑOL COMO LENGUA EXTRANJERA*
}

\author{
ORAL CORPUS FOR THE STUDY OF THE ACQUISITION AND \\ LEARNING OF THE PHONIC COMPONENT OF SPANISH AS A \\ FOREIGN LANGUAGE
}

\author{
ANA BLANCO CANALES \\ Universidad de Alcalá. Alcalá de Henares, España. \\ ana.blanco@uah.es
}

\section{RESUMEN}

La falta de atención prestada al componente fónico en la enseñanza de español como lengua extranjera repercute decisivamente en un desarrollo deficitario e incompleto de la competencia comunicativa de los alumnos, habida cuenta de la importancia de este componente para las destrezas orales (expresiva, auditiva y de interacción) y de sus múltiples interrelaciones con las demás subcompetencias (lingüísticas, pragmática y sociolingüística). Al mismo tiempo, el propio proceso de aprendizaje se ve enturbiado como consecuencia de la ansiedad y falta de confianza que supone no dominar los elementos fónicos de la lengua que se estudia. Para poder hacer frente a esta situación se requiere contar con materiales orales y herramientas de análisis con los que se puede desarrollar estudios de distinta naturaleza sobre la adquisición y aprendizaje del componente fónico, cuyos resultados puedan aplicarse o transferirse a la mejora de la enseñanza, a la elaboración de materiales didácticos, a la programación curricular y diseño de cursos y a la formación de profesores. Esto son los objetivos del corpus AACFELE; a lo largo de estas páginas mostraremos su diseño, sus características y la metodología de trabajo que se ha seguido en su elaboración.

Palabras clave: Corpus oral, componente fónico, español como lengua extranjera.

* Este trabajo se inscribe dentro del proyecto Adquisición y aprendizaje del componente fónico del español como lengua extranjera / segunda lengua (ref. FFI2010-21034), financiado por el Ministerio de Ciencia e Innovación de España. 


\section{ABSTRACT}

The lack of attention to the phonics component in teaching Spanish as a foreign language decisively impacts the communicative competence of students, in terms of oral expression, comprehension and interactive skills and other related sub-competence (linguistic, pragmatic and sociolinguistic). With regard to students, both anxiety and lack of confidence in their ability to pronounce also cloud their process of learning. This situation, therefore, requires compiling oral materials and analysis tools aimed at improving studies on the acquisition and learning of the phonics component. The results obtained from these studies can be used to improve teaching, develop training materials, program and design curriculums, and train teachers. These are the objectives of the AACFELE corpus. Throughout, the purpose of this article is to present the design, characteristics and methodology of the AACFELE corpus.

Keywords: Oral corpus, phonetic component, Spanish as a foreign language.

Recibido: 12.03.2012. Aceptado: 11.07.2012.

\section{LA COMPETENCIA FÓNICA}

L a competencia fónica es la capacidad que permite a un individuo producir y reconocer los elementos propios de una lengua a todos los niveles (sonidos, unidades rítmicas, unidades entonativas) y, al mismo tiempo, le permite identificar los que no lo son (Iruela, 2004: 35). Según el Marco común europeo de referencia para las lenguas: aprendizaje, enseñanza, evaluación (2002: epígrafe 5.2.1.4; en adelante, $M C E R$ ) se puede definir como la destreza en la percepción y la producción de: a) las unidades de la lengua (fonemas) y su realización en sonidos concretos (alófonos); b) los rasgos fonéticos que distinguen a los fonemas entre sí (por ejemplo: sonoridad, nasalidad, oclusión, labialidad); c) la composición fonética de las palabras (estructura silábica, la secuencia acentual de las palabras, etc.); d) los fenómenos de coarticulación (asimilación al punto de articulación, reducción de las vocales átonas, relajación articulatoria, elisión de sonidos consonánticos); e) acento y el ritmo de las oraciones; f) la entonación.

Tras algunas décadas de abandono como consecuencia del énfasis puesto en una mal entendida "comunicabilidad" del lenguaje, estudios recientes sobre la competencia fónica coinciden en considerarla una parte fundamental de las destrezas orales de la lengua, esto es, de la expresión oral, la comprensión auditiva y la interacción oral (Iruela, 2007). Por lo que respecta a la expresión oral, debemos partir del hecho, evidente, de que la pronunciación es el medio a través del cual se transmite la información y, por lo tanto, la comprensión o no del mensaje por parte del oyente va a depender de la calidad de esa transmisión. Pero sería una visión bastante 
reduccionista considerarla únicamente como un medio, pues la pronunciación no solo es portadora del contenido léxico-semántico de las unidades, sino que, en sí misma, aporta valor comunicativo de enorme importancia al mensaje (transmite actitudes, intenciones, información sociocultural, contenidos expresivos).

En cuanto a la comprensión auditiva, la competencia fónica desempeña también un papel decisivo, dado que uno de los procesos que conforman esta destreza es el de la percepción de los elementos fónicos ${ }^{1}$. Si el hablante no identifica correctamente los segmentos que componen un texto oral, tendrá dificultades para comprenderlo e interpretarlo. Esto implica, entonces, que existe una relación directa entre capacidad para percibir y discriminar elementos fónicos de la L2 (los contrastes fonológicos, los sonidos que representan a cada fonema, el ritmo, la entonación, el acento a nivel de palabra y de frase, etc.) y la mayor facilidad para la comprensión del mensaje. Podemos afirmar, por tanto, que un nivel de competencia fónica insuficiente es un factor que dificulta la tarea de la comprensión auditiva.

La pronunciación forma parte de la subcompetencia lingüística; es, por ello, una de las numerosas piezas que integran la competencia comunicativa (Hymes, 1972; Canale y Swain, 1980; Bachman, 1995). Como el resto de las piezas, no se halla aislada de las demás ni actúa en solitario, sino que participa del entramado de interrelaciones y zonas de intersección que definen la competencia comunicativa. Con respecto a la competencia lingüística, la pronunciación interviene de manera decisiva en el buen desarrollo de las subcompetencias léxica y gramatical. Por una parte, la pronunciación se relaciona con el vocabulario en tanto su adquisición implica, entre otras cosas, su correcta percepción y producción en la lengua oral. Dominar el sistema fónico de la L2 facilita la adquisición del vocabulario, pues permite su mejor almacenamiento (la palabra se recuerda por su representación fónica) así como un uso eficaz (Linell, 1982; Ellis, 1996; Hu, 2003).

Por otra parte, la pronunciación afecta al uso gramatical en tres aspectos concretos: la morfología, la sintaxis y el ritmo de las palabras átonas (Iruela, 2004). Una competencia fónica deficitaria puede llevar a generar errores en la distinción singular/plural, presente de indicativo/presente de subjuntivo (canta/cante), primera/tercera persona del singular ( he ido/ha ido), segunda/tercera persona del singular (cantas/canta), futuro/imperfecto del subjuntivo (cantarálcantara), pretérito perfecto simple/presente de subjuntivo (cantélcante). Ocurre en muchas ocasiones que este tipo de errores se interpreta como un problema gramatical (con su consecuente corrección y confusión en los alumnos) cuando, en realidad, se trata de un defecto fónico. En cuanto a la sintaxis, es de todos conocida la estrecha relación entre tipo y estructura oracional y curva de entonación (una línea melódica inade-

${ }^{1}$ Otros procesos son: anticipar qué se va a escuchar, percibir los elementos fónicos del enunciado, identificar usando la competencia lingüística, comprender desde el punto de vista semántico, inferir los vacíos de significado o interpretar el significado según el contexto. 
cuada entorpece sobremanera la interpretación del enunciado). Hasta tal punto esta relación es importante, que son muchos los estudios que demuestran que el principal problema fónico en aprendizaje de una lengua extranjera es el de la entonación, ya que, en este caso, afecta a unidades de comunicación, de transmisión de significados. Por último, con respecto a las palabras átonas, su correcta pronunciación requiere que sean articuladas junto a una tónica. Si se emiten separadamente, se transforman en tónicas, lo que conlleva, en muchos casos, cambio de categoría gramatical e interpretación referencial errónea.

Centrémonos ahora en la relación que se da entre la competencia fónica y la pragmática. El valor funcional de los enunciados está muy ligado a la entonación y la intensidad, que son los elementos que transmiten la verdadera actitud o intención del hablante (pregunta, indiferencia, sarcasmo, ironía, sorpresa). De hecho, en aquellos casos de discordancia entre el valor literal del enunciado y el de la entonación, prevalece el de esta última. La entonación, es por ello, un elemento altamente comunicativo, pues es portador de función, intención y significado.

Finalmente, cabe mencionar que la pronunciación interviene decisivamente en la competencia sociolingüística, ya que ésta proporciona información sobre nuestra persona (origen geográfico, edad, nivel sociocultural) y sobre el contexto y la situación en los que tiene lugar la comunicación. Como señalan Dieling y Hirschfeld (2000),se puede decir que es nuestra imagen ante los demás. En este sentido, una mala pronunciación o un fuerte acento extranjero pueden provocar una actitud negativa en los interlocutores, dado que esa información sobre la identidad social y geográfica que aportan puede ser transmisora de prejuicios y estereotipos (Munro, 2003; Derwing, Rossiter, Munro y Thomson, 2004).

Como vemos, la compentencia fónica no puede, en la actualidad, situarse al margen de una visión global e integradora de la lengua en la que todos los fenómenos están interrelacionados y son igualmente decisivos para lograr un uso eficaz y adecuado.

\section{SOBRE LA ENSEÑANZA/APRENDIZAJE DEL COMPONENTE FÓNICO}

La pronunciación defectuosa en una lengua extranjera no es sólo un indicador de bajo nivel de conocimiento de la lengua; es, sobre todo, un problema de comunicación y, por ello mismo, de interrelación social. Las deficiencias fónicas llegan, en muchas ocasiones, a dificultar la interacción y hacer inteligible el mensaje. Pero sin llegar a casos tan extremos, se puede señalar que las dificultades para entender a un hablante no nativo suelen ser la causa que más incide en su aislamiento y en la ausencia de comunicación e integración en un medio lingüístico. Mientras que los errores gramaticales, léxicos o, incluso, pragmáticos raras veces producen malestar 
o incomodidad en el interlocutor, que acaba neutralizando parte de esos errores y ayudando a corregir otros, los problemas fónicos llegan a desencadenar un efecto evitación: "si no logro entender a una persona, la evito en lo posible". El propio acento extranjero puede traer consigo consecuencias negativas, ya que, además de reducir la inteligibilidad o la correcta interpretación, podría llevar a una evaluación social negativa e incluso, en algunos casos, a la discriminación (Lippi-Green, 1997; Munro, 2003), al estar fuertemente asociado a la identidad (Dauer, 2005). Por lo tanto, cuando hablamos de adquisición y aprendizaje del componente fónico, no estamos simplemente aludiendo a un hecho de corrección y perfeccionamiento lingüístico. Hablamos de comunicación en sociedad, de interacción y de integración dentro de una comunidad o de un grupo.

Todo ello permite afirmar que la adquisición de una lengua extranjera debe comenzar, inevitablemente, por el nivel fónico. Aunque existe un acuerdo general sobre la relevancia de la pronunciación en enseñanza y aprendizaje de lenguas segundas y extranjeras, sin embargo ocupa un lugar secundario en los programas académicos (Moreno, 2002); tampoco en la práctica cotidiana del aula se suele prestar demasiada atención a los problemas fónicos de los estudiantes (Poch, 2004a). A esto podría añadirse que son muy pocos los trabajos de didáctica centrados en este aspecto de la expresión oral, tanto teóricos como prácticos. Así, pues, aunque no se pone en duda la importancia de la enseñanza de la pronunciación para el aprendizaje de una lengua extranjera, así como para una comunicación eficaz, y a pesar de que todos los implicados (alumnos, profesores, programadores, autores de materiales) son conscientes de ello, la realidad es que sigue ocupando un lugar ínfimo tanto en los programas docentes como en los manuales, como en la actividad del profesor.

Las razones de esta contradicción son varias. Por una parte, habría que mencionar que los propios enfoques metodológicos no conceden la misma importancia a los contenidos fonético-fonológicos que a los demás. La atención que se le presta queda reducida en el tiempo al periodo que el alumno necesite hasta lograr comunicarse (Poch, 2004b). Es decir, que el interés por la pronunciación desaparece en el momento en que deja de interferir en la comprensión de los enunciados. Sin embargo, distintas investigaciones han demostrado que la enseñanza sistemática de la pronunciación es beneficiosa para la adquisición de la lengua no sólo porque favorece la comprensión de los enunciados y facilita la expresión, sino también porque disminuye en el alumno su sensación de ansiedad con respecto a la comunicación oral (Elliott, 1997).

La creencia tan extendida de que la imitación es suficiente para aprender los aspectos fonéticos de una lengua contribuye, igualmente, a que se reste importancia a la enseñanza de la pronunciación. Otra de las razones que explica el abandono de estos contenidos es la falta de formación específica de los profesores. Normalmente, éstos han recibido formación teórica en fonética y fonología del español, 
pero no práctica, al menos, aplicada a la enseñanza. En otros casos -contenidos gramaticales, léxicos, funcionales, culturales, etc.- la propia experiencia docente va formando al profesor, pero en éste esa autoformación es muy difícil, dada la naturaleza de la propia materia y la falta de herramientas para un autoaprendizaje eficaz. Por otra parte, en los programas formativos para profesores de español es poco o nulo el tiempo que se dedica a trabajar la cuestión de la pronunciación; asimismo, se aprecia con facilidad que hay menos investigación y menos especialistas en esta área que en otras relacionadas con la enseñanza del español. En realidad, se trata de un área de trabajo muy poco transitada. En la mayor parte de las ocasiones, lo que subyace a estas razones es simplemente la falta de material con el que ir formándose en aspectos de fonética, con el que proporcionar una formación práctica y aplicada, con el que poder desarrollar trabajos de investigación, o que sirvan como orientación para los autores de materiales didácticos.

Habría que añadir otras razones, como es la falsa creencia de que el español es una lengua cuyo componente fónico es simple y que, por tanto, no merece la pena entretenerse demasiado en cuestiones que los alumnos pueden "aprender por sí mismos"; o la idea de que es necesario ser un especialista en fonética para trabajar con los alumnos este componente lingüístico de manera sistemática. Con respecto a lo primero, basta con escuchar atentamente a hablantes no nativos, incluso de niveles de lengua altos (B2, C1), para darnos cuenta de la gran cantidad de errores de pronunciación que caracterizan su discurso y de las muchas dificultades articulatorias y prosódicas a las que se van enfrentando a cada instante. En cuanto a la segunda cuestión, es suficiente con realizar algunos análisis fónicos (análisis dirigidos, más o menos guiados) para percatarnos de que, sin ser especialistas, podemos llegar a tener una idea certera del repertorio de errores de un estudiante $y$, por tanto, de sus necesidades de aprendizaje.

\section{EL CORPUS AACFELE}

El corpus $A A C F E L E$ es uno de los resultados fundamentales del proyecto Adquisición y aprendizaje del componente fónico del español como lengua extranjera / segunda lengua, financiado por el Ministerio de Ciencia e Innovación, dentro del Plan Nacional I+D+i (Ref. FFI2010-21034). El proyecto, liderado por la Universidad de Alcalá, reúne a investigadores de otras ocho universidades (La Laguna, Kapodistríaka de Atenas, Fu Jen Catholic University, Tamkang University, Universidad de Paderborn, Universidad de El Cairo, Universidad de Silesia, Universidad de Bragança) y tiene como objetivo fundamental proporcionar el material, las herramientas y los instrumentos necesarios para facilitar el desarrollo de estudios sobre la adquisición y aprendizaje de la fonética del español como lengua extranjera desde diferentes orientaciones (análisis contrastivos, impacto social de las deficiencias 
fónicas, influencia de variables sociolingüísticas, propuestas de actuación, análisis del proceso de adquisición). Al mismo tiempo, pretende facilitar a profesores y autores de materiales información, datos y ejemplos sobre los aspectos que se deben trabajar en el aula en función de las características de su grupo, así como ofrecerles la oportunidad de ir autoformándose en este campo de trabajo de una manera práctica y útil, a través de la propia experiencia del análisis fonético. El proyecto quiere cumplir también la misión de servir de guía y orientación a los responsables de elaborar programas académicos o marcos curriculares. En este amplio contexto de trabajo, el corpus constituye el eje principal del proyecto y el elemento que permitirá ir satisfaciendo los objetivos que se han planteado.

\subsection{Descripción, objetivos, acceso}

El corpus $A A C F E L E$ está actualmente compuesto por muestras de habla procedentes de estudiantes alemanes, griegos, taiwaneses, polacos, portugueses y egipcios que se hallan en posesión de los niveles A2, B1, B2 y C1 de español, según el MCER. En fases posteriores, se pretende ir ampliando tanto las nacionalidades presentes como los niveles lingüísticos de los informantes.

En consonancia con los objetivos generales del proyecto en el que se inscribe, el corpus tiene como finalidad principal facilitar el desarrollo de algunas líneas de investigación, tanto de carácter teórico como aplicado, sobre adquisición y aprendizaje del componente fónico del español como lengua extranjera. Con ello, se pretende, a su vez, contribuir a la mejora de la enseñanza de la pronunciación del español y, por ende, a la enseñanza del español en general, habida cuenta de la importancia y de la interrelación de este componente con los demás y con las destrezas comunicativas.

De manera más concreta, el corpus persigue proporcionar muestras de lengua y las herramientas necesarias para el manejo, la búsqueda y la cuantificación de los datos, lo que facilitará el desarrollo de esos trabajos de investigación, entre los que ocuparán un lugar destacado el del análisis de los errores atendiendo tanto a factores lingüísticos (lengua materna, nivel de conocimientos, registro) como a otros de tipo sociocultural (nacionalidad, edad, sexo, nivel formativo, relación con el país extranjero...).

Tanto el corpus como las herramientas asociadas se hallan a disposición de los interesados en la página web del proyecto: http://www2.uah.es/fonoele.

\subsection{Características}

Una de las fases más complejas en la construcción de un corpus de datos orales, como señala Moreno (1990: 78), es la de selección cualitativa y cuantitativa de los informantes, fase que nos lleva necesariamente a reflexionar sobre aspectos 
teóricos de los que partimos (en nuestro caso, validez de los niveles universales de lengua; concepto de lengua materna o lengua extranjera, etc.) y sobre aspectos metodológicos (representatividad, tipos de muestras, etc.). La primera cuestión a la que debemos hacer frente es la del número de informantes con los que elaboraremos la muestra. Su representatividad está en relación con la población que se quiere describir, lo que requiere identificar y delimitar dicha población. En este caso, se trata de un universo de estudio tremendamente heterogéneo y muy difícil de acotar a partir de una serie de especificaciones, pues lo único que constituye un elemento válido de descripción general es el hecho de que son estudiantes de español. Esto nos ha llevado a considerar seis poblaciones diferentes, que surgen por la nacionalidad de los estudiantes. Hemos elaborado para cada caso una muestra específica, aunque de características comunes.

De las diferentes técnicas para la preparación de la muestra (muestreo estratificado al azar, agrupado o en racimo, accidental, etc.) nos hemos decantado por muestreos de no probabilidad, concretamente, por los conocidos como muestreo por cuotas e intencional. Consiste en dividir la población en grupos y buscar informantes para cada uno de ellos, de manera que todos los grupos estén representados. Estos son seleccionados por el investigador de acuerdo al cumplimiento de unos requisitos establecidos.

Con respecto al número de informantes, no existen datos contrastados que indiquen, para un estudio de estas características, qué cantidad resulta representativa, si bien sabemos que los comportamientos lingüísticos son bastante más homogéneos que los sociales (Labov, 1996), característica que se acentúa en el caso de las interlenguas, dadas las escasas posibilidades que tienen los alumnos de hacer un uso creativo, original o diferenciador de sus recursos. Si pensamos, además, que analizaremos realizaciones fónicas, las posibilidades disminuyen aún más, como sucede con las propias lenguas maternas. Tras revisar la bibliografía al respecto, finalmente consideramos que utilizaríamos para cada submuestra un total de 16 locutores, lo que nos ha conducido a constituir un corpus inicial con 96 informantes.

Dada la naturaleza del corpus (estudiantes de español de diferentes países y con distintos niveles de lengua), hemos tratado de homogeneizar las características de los locutores y evitar, así, diferencias entre unos países y otros, con objeto de que los datos sean susceptibles de comparación. No obstante, como nos interesaba estudiar los procesos de adquisición, aprendizaje y enseñanza del componente fónico atendiendo a algunos de los parámetros, que según la bibliografía son relevantes o intervienen en alguna medida, hemos intentado manejar aquellas variables que pueden resultar significativas, pero desechar aquellos otros rasgos que vienen a complicar más aún la variedad implícita y que no aportan resultados pertinentes para nuestro proyecto. Para el establecimiento de la muestra se tuvieron en cuenta, finalmente, factores sociales y factores lingüísticos externos: 
Factores sociales:

- sexo

- edad

- contacto con el español

Factores lingüísticos externos

- nivel de lengua

- segunda lengua extranjera

- experiencias de aprendizaje

\subsubsection{Características sociales de los locutores}

En esta primera fase han participado en las grabaciones estudiantes de español de nacionalidad taiwanesa, alemana, griega, portuguesa, polaca y egipcia. Como ya se ha señalado más arriba, contamos, para cada caso, con 16 locutores, repartidos a partes iguales entre hombres y mujeres, aun a sabiendas de que son muchas más las mujeres que estudian lenguas extranjeras y, especialmente, español.

Algunos autores, como Altman (1980), consideran el sexo como uno de los muchos factores que condicionan el proceso de aprendizaje de una lengua extranjera, junto con la edad, las experiencias previas de aprendizaje, la actitud, la motivación, la personalidad, etc., aunque no se encuentra entre los factores más recurrentes en los estudios. A pesar de ello, hemos querido recogerlo en nuestro corpus, pues al tratarse de adultos, nos parecía posible que se apreciara un comportamiento lingüístico diferente entre hombres y mujeres, de la misma manera que ocurre en lengua materna, como numerosísimos estudios sociolingüísticos lo demuestran (García Moutón, 2000). Nos resultaba de sumo interés comprobar si, también en una segunda lengua, las mujeres se muestran más sensibles a la norma de referencia o a la corrección, lo que vendría a indicar que han desarrollado una mayor conciencia lingüística en su interlengua. Nuestro interés por este parámetro de variación responde más a intereses sociolingüísticos que educativos; dada la edad de los aprendices, no parece que sean ya muy significativas las diferencias que, en cambio, sí se observan en ámbitos formales de educación, entre niños y niñas en edades tempranas, reflejo de las distintas etapas de maduración cognitiva entre unos y otras (véase Cano García, 2000; Howard, 1999).

En cuanto a la edad, es un factor que ha demostrado ser crucial en el desarrollo del componente fónico en una lengua extranjera, pues muchos estudios han comprobado la existencia de diferencias significativas entre la pronunciación que alcanzan los niños y la que alcanzan los adultos (por ejemplo, Patkowski, 1990), lo que ha conducido a afirmar que siguen procesos de adquisición/aprendizaje diferentes. Lo que no resulta tan claro de explicar es en qué consisten los cambios que implica la edad, por qué suceden y cuándo. 
Lenneberg (1967) propuso la existencia de un periodo crítico para la adquisición de una L2 predeterminado biológicamente, tras el cual es imposible una adquisición completa, exenta de acento extranjero. Para Scovel (1969) este periodo crítico sólo afecta a la pronunciación, pues es el único aspecto lingüístico con una base neuromuscular. Otros autores mantienen una postura algo menos radical y hablan de un periodo de mayor sensibilidad durante el cual las condiciones para la adquisición de una lengua concreta son óptimas. El final del periodo no sucede de forma brusca, sino que se da paulatinamente, y discurre en paralelo a la progresiva pérdida de la capacidad de aprendizaje, de manera que cuanto más alejado en el tiempo, mayores dificultades para lograr buenos resultados. Aunque no existe unanimidad, sin embargo, buena parte de los autores que defienden la existencia de este periodo sensible señalan su finalización antes de los 15 años (Long, 1990, lo sitúa a los 6 años; Johnson y Newport, 1989, a los 7 años; Scovel, 1988, en torno a los 10; Oyama, 1976, sobre los 12; Patkowski, 1990, antes de los 15).

Aunque en minoría, hay autores que descartan la edad como un factor determinante para la adquisición del componente fónico. Así, Flege $(1987,1991)$ o Cantero (1997), por ejemplo, defienden que las diferencias observadas en función de la edad responden más a factores socioafectivos, contextuales, ambientales, educacionales, etc., que a los cambios biológicos o neuronales del individuo.

Para nuestro trabajo, la edad resulta un factor interesante ya que nos va a permitir analizar si el proceso de pérdida de la capacidad para el aprendizaje de la pronunciación concluye en un momento más o menos definido de la vida del individuo o si, por el contrario, se da a lo largo de toda su existencia, de manera que las dificultades para la adquisición del componente fónico aumentan proporcionalmente a la edad. Según este planteamiento, deberían observarse diferencias entre los dos grupos de edad con los que decidimos trabajar. De no apreciarse así, podríamos afirmar que en edad anterior a los 18 ha concluido definitivamente ese periodo sensible del que hablan muchos autores, con lo cual las posibilidades de un progreso efectivo son escasas. Pero si existen esas diferencias es porque permanece la capacidad, aunque disminuida, lo que podría llevarnos a pensar que un entrenamiento adecuado puede frenar o aminorar los efectos del proceso de pérdida de la capacidad.

Decidimos trabajar en esta primera fase con dos grupos de edad: grupo 1, de 18 a 25 años y grupo 2, de 26 a 35 años. Puesto que uno de los objetivos fundamentales es el establecimiento de los errores estandarizados según la nacionalidad, trabajar con hablantes menores de 16 años implica tener que atender, necesariamente, a cuestiones relacionadas con los procesos de fijación paramétrica, todavía en curso -aunque en sus fases finales-, lo que significaba que las posibilidades de interpretación del error ascenderían de manera notable, así como las de cruce entre fenómenos propios de la lengua materna y fenómenos de la lengua extranjera. Con respecto a hablantes de edad superior a 35, se trata simplemente de un límite, pues, hasta los 50, las diferencias no son apreciables. Sin embargo, a partir 
de esa edad, como consecuencia de los cambios hormonales que experimenta el cuerpo, la voz (y el sonido articulado) sufre cambios notables. Es importante señalar que, en todos los casos, seleccionamos alumnos que cursaban sus estudios de español en el momento de la recogida de datos y que no llevaban más de cinco años como aprendices de nuestra lengua. Decidimos descartar a posibles locutores que estudiaron español de niños o adolescentes, porque el paso del tiempo podría introducir factores de variación que no estábamos controlando.

El tercer factor que hemos tenido en consideración es el que hemos denominado contacto con el español. Se trata de un parámetro de enorme complejidad por la dificultad que ha planteado su establecimiento. Se han diferenciado cuatro grados distintos de contacto: alto, medio, bajo o muy bajo. La adscripción de un locutor a un nivel concreto se realiza a partir de la puntuación media obtenida en dos dimensiones: viajes y vida cotidiana. Ambas variables vienen determinadas, a su vez, por tres factores, como se ve en la Tabla I:

Tabla I. Factores que definen la variable contacto con el español.

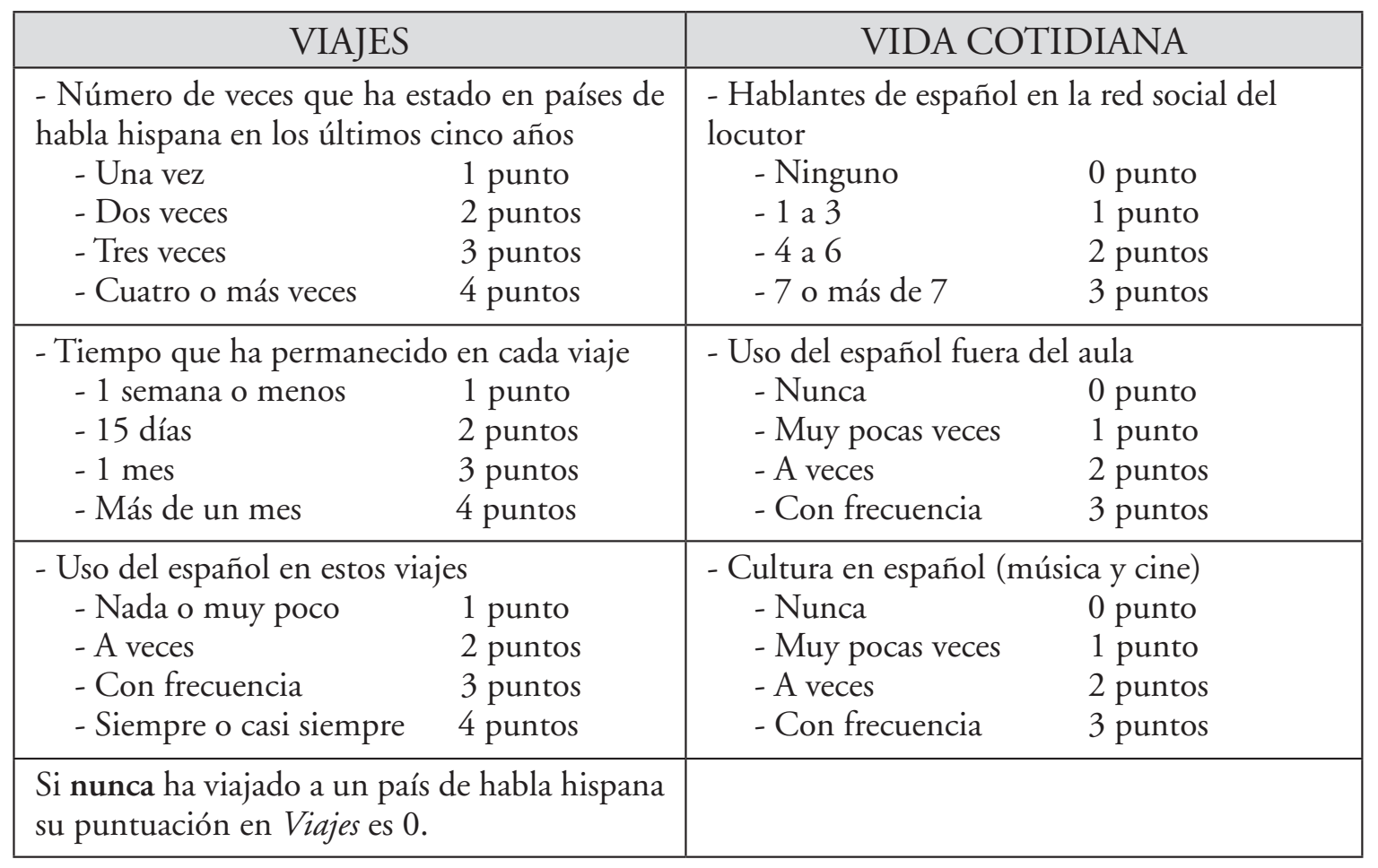

Para obtener un grado alto de contacto con el español, el locutor debe lograr entre 19 y 24 puntos; en el caso de medio, entre 13 y 18 puntos; el grado bajo se adquiere cuando se obtiene entre 7 y 12 puntos y, por último, el grado muy bajo, cuando se está entre 0 y 6 puntos. Nos ha parecido conveniente distinguir entre bajo y muy bajo porque recoge un abanico de diferentes situaciones con las que nos podemos encontrar. Una de las alternativas hubiera sido considerar sólo la opción bajo, pero dejábamos fuera a aquellos estudiantes (muchos) cuyos contactos 
con el español se limitan a escuchar algo de música, ver alguna película de vez en cuando o a participar ocasionalmente en alguna red social; la otra alternativa era diferenciar entre bajo y nada, pero, nada significa 'cero, ausencia absoluta' y las posibilidades de encontrar estudiantes ajenos absolutamente a la realidad hispana son mínimas. Además, esto nos llevaba de nuevo a desentendernos de ese gran grupo que mantiene escasos contactos.

A diferencia de las variables edad y el sexo, en las que se buscó expresamente un número determinado para cada variante, en este caso, si bien tratamos de que hubiera locutores para cada casilla, no hubo una distribución premeditada, sino que fue producto del azar.

\subsubsection{Características lingüisticas de los locutores}

Hemos tratado de que, en cada una de las submuestras (estudiantes alemanes, polacos...), los locutores tuvieran una única lengua materna y que ésta fuera la misma para todos (la lengua oficial del país o la variedad de prestigio mayoritaria). Hubiera sido muy interesante atender a la influencia en el desarrollo de la competencia fónica de factores como el bilingüismo (en sus diversas modalidades), el tipo de lengua materna y su relación con la lengua de cultura (en los hablantes bilingües), etc., pero habría dificultado enormemente la puesta en marcha del proyecto. De la misma manera, hemos perseguido que todos los estudiantes poseyeran la misma variedad dialectal, pues la diversidad geográfica podría arrojar interpretaciones erróneas de muchos fenómenos. Puesto que no hemos podido elegir la variedad, en cada caso ha sido aquella predominante en el lugar en el que se encontraba el investigador que recogió los datos. Por lo que respecta a las posibles variedades sociolectales, también en este caso hemos creído conveniente unificar al máximo para aminorar los efectos de la diversidad social natural de cada comunidad. Además, no nos pareció interesante atender a diferencias socioeconómicas o socioculturales, por ejemplo, dado que las categorías que fijáramos no serían comparables de unos países a otros. Por otra parte, para los objetivos de nuestro proyecto no resultaban relevantes.

En cuanto a la(s) lengua(s) extranjera(s) que hablan o estudian los locutores, hemos buscado, de un lado, homogeneidad con respecto a la primera lengua (en todos los casos, tenía que ser el inglés), y de otro, heterogeneidad en cuanto a la segunda y tercera lengua. Concretamente, lo que nos interesaba era conocer qué posición ocupa el español en su repertorio de lenguas extranjeras y la relación de parentesco entre ellas. No obstante, este último aspecto no se ha tenido en cuenta para el establecimiento de la muestra y, consecuentemente, no aparece como variable de covariación en los análisis cuantitativos, ya que las posibilidades eran muy numerosas, lo que imposibilitaba contar con una mínima representación para cada caso. Sin embargo, sí resultará información de sumo interés a la hora de 
analizar e interpretar muchos de los resultados obtenidos y, previsiblemente, podrá ayudarnos a entender algunos fenómenos.

El primer factor lingüístico externo que manejamos fue el del nivel de lengua. Esta variable quedó divida en cuatro variantes, A2, B1, B2 y C1, que corresponden a cuatro de los seis niveles que establece el MCER. Decidimos sacar de la muestra a los alumnos de A1, dado su escaso conocimiento de la lengua (están en una fase incipiente de aprendizaje, en un primer contacto), y a los de C2, por tratarse de hablantes definidos por el MCER como bilingües. La asignación del locutor al nivel correspondiente se ha realizado en dos fases: en la primera, la decisión ha recaído en el investigador responsable de realizar las grabaciones, para lo cual ha seguido muy de cerca los descriptores del $M C E R$, así como las especificaciones consensuadas por todo el equipo. En la segunda, una comisión de expertos, formada por profesores con larga experiencia en acreditación de nivel, ratificó la asignación o propuso otra distinta. En la mayoría de los casos ha habido coincidencia entre el punto de vista del investigador y el de la comisión; en los casos de desacuerdo, ha prevalecido el criterio de la comisión, dado que gozaba de una visión global de la que carecía cada uno de los miembros del equipo. Pese a no tratarse éste de un procedimiento estandarizado, nos pareció el más fiable, pues seguir criterios como los años de estudios de español, la clasificación que realice el propio centro de estudios, una prueba de nivel, etc., generarían una enorme disparidad. El número de estudiantes por nivel fue una decisión previa y estuvo motivada por la relación proporcional aproximada con el número total de alumnos para cada caso. Se acordó, para cada nacionalidad, el siguiente reparto:

- Nivel A2: 6 locutores

- Nivel B1: 6 locutores

- Nivel B2: 2 locutores

- Nivel C1: 2 locutores

Finalmente, tuvimos en consideración el factor experiencias de aprendizaje, con objeto de valorar la incidencia del trabajo sistemático de la fonética sobre la calidad de la pronunciación. Se contemplaron tres grados:

- Habitualmente ha trabajado/trabaja en el aula aspectos fónicos.

- En algunas ocasiones ha trabajado/trabaja en el aula aspectos fónicos.

- Nunca o casi nunca ha trabajado/trabaja en el aula aspectos fónicos.

En principio, cabría esperar que aquellos estudiantes con un adiestramiento continuado en cuestiones fónicas presenten menos dificultades de pronunciación. Sin embargo, algunos autores (Torres, 2001) sostienen que, dada la fuerte dependencia de la corrección fónica respecto a la lengua escrita (la mayor parte de las ac- 
tividades con las que se practica son de base escrita), la formación resulta de escasa o nula eficacia. En este mismo sentido se manifiesta Cantero (1997), quien afirma que la fuerte base lectoescritura de la enseñanza de lenguas extranjeras es la causa del mayor retraso del desarrollo de habilidades auditivas y expresivas. Efectivamente, hay algunos tipos de errores fónicos que tienen su base exclusivamente en la ortografía, y que no existirían si no se tuviera como referencia la escritura. Y más allá del nivel fónico, se podría afirmar que problemas como la falta de fluidez, la inexpresividad, el escaso desarrollo de estrategias discursivas, etc., son achacables a la presión de la lengua escrita, pues es el modelo fundamental al que es expuesto el alumno y el que tiene siempre como referencia.

\subsection{Datos recogidos}

\subsubsection{Material lingüistico de estudio}

El corpus $A A C F E L E$ tiene por objeto conseguir datos suficientes, desde un punto de vista de representatividad estadística, con los que poder a) describir con exhaustividad los errores asociados a la adquisición del componente fónico de español como lengua extranjera, b) comprender bien los problemas para su aprendizaje y c) establecer pautas para la mejora de la enseñanza, para la corrección, etc. Por esta razón, se ha tratado de que, en el diseño del material lingüístico objeto de estudio, esté presente el mayor número posible de elementos fónicos del español, pretensión que, como es fácil de adivinar, ha estado muy limitada por razones de tiempo, recursos, etc. En este sentido, hemos tenido que realizar un gran esfuerzo para reducir los infinitos elementos que constituyen el universo fónico de nuestra lengua a un número finito y manejable. Esto nos ha llevado a desechar todas las realizaciones dialectales, sociolectales y estilísticas para mantenernos en el plano más abstracto de las variantes alofónicas.

Los fenómenos que se analizarán pertenecen tanto al nivel segmental (sonidos y grupos de sonidos, incluidos en este último caso los que se generan por fonética sintáctica) como suprasegmental (intensidad, grupos fónicos y entonación). En ambos casos, el repertorio de elementos está ampliamente descrito en la bibliografía, a excepción de la entonación, dado que los estudios destinados a describir e inventariar todas las posibilidades expresivas del español son escasos y discordantes. Es natural esta situación si tenemos en cuenta que se trata de un fenómeno que aglutina rasgos fónicos, sintácticos, textuales, pragmáticos y sociolingüísticos. Nuestro punto de partida ha sido la propuesta de los ocho patrones tonales básicos de Cantero $(2002)^{2}$, que para nuestra investigación hemos reducido a seis, dada

${ }^{2}$ Estos ocho patrones son el resultado de combinar los tres rasgos verdaderamente fonológicos que, según Cantero, describen la entonación: [+/- interrogación], [+/- énfasis] y [+/- suspensión]. 
la escasa probabilidad de aparición de los tonemas [+ interrogativo, + enfático, + suspensión] y [+ interrogativo, - enfático, + suspensión]. Hemos tratado de buscar diferentes modelos sintácticos para cada tonema considerado.

Puesto que en la pronunciación de una lengua los sonidos no aparecen aislados, sino en contacto unos con otros, permanentemente influidos y modificados por los vecinos, el estudio de cada uno debe tratar de atender a las posibilidades combinatorias más frecuentes o de mayor relevancia en esa lengua. En numerosas ocasiones, la dificultad articulatoria no estriba tanto en la emisión de ese sonido en estado "puro" como en su pronunciación en contexto (coarticulación). Esta circunstancia es de especial importancia en el aprendizaje de una lengua extranjera para poder orientar la práctica, la corrección y la enseñanza de la manera adecuada. Así, en nuestro corpus, los instrumentos para la recogida y análisis de datos se han diseñado de forma que permitan estudiar la incidencia de diferentes rasgos fónicos en la emisión de un elemento concreto.

El repertorio de unidades objeto de estudio junto con sus factores de incidencia se muestra a continuación:

1. Vocales. Objeto de estudio: 10 alófonos vocálicos (5 orales y 5 nasales).

\begin{tabular}{|c|c|}
\hline $\begin{array}{l}\text { 1. Intensidad } \\
\text { Vocal átona } \\
\text { Vocal tónica }\end{array}$ & $\begin{array}{r}\text { 2. Estructura silábica } \\
\text { Sílaba abierta } \\
\text { Sílaba cerrada }\end{array}$ \\
\hline $\begin{array}{l}\text { 3. Punto de articulación de la consonante } \\
\text { anterior } \\
\qquad \begin{array}{l}\text { Labial }^{3} \\
\text { Dental } \\
\text { Alveolar } \\
\text { Velar } \\
\text { Palatal }\end{array}\end{array}$ & $\begin{array}{l}\text { 4. Punto de articulación de la consonante } \\
\text { posterior } \\
\qquad \begin{array}{l}\text { Bilabial } \\
\text { Alveolar } \\
\text { Velar }\end{array}\end{array}$ \\
\hline $\begin{array}{l}\text { 5. Modo de articulación de la consonante } \\
\text { anterior } \\
\text { Oclusiva } \\
\text { Fricativa } \\
\text { Nasal } \\
\text { Vibrante } \\
\text { Lateral }\end{array}$ & $\begin{array}{l}\text { 6. Modo de articulación de la consonante } \\
\text { posterior } \\
\text { Oclusiva } \\
\text { Fricativa } \\
\text { Nasal } \\
\text { Vibrante } \\
\text { Lateral }\end{array}$ \\
\hline $\begin{array}{l}\text { 7. Vibración laríngea de la consonante } \\
\text { anterior } \\
\text { Sorda } \\
\text { Sonora }\end{array}$ & $\begin{array}{l}\text { 8.Vibración laríngea de la consonante } \\
\text { posterior } \\
\text { Sorda } \\
\text { Sonora }\end{array}$ \\
\hline
\end{tabular}

\footnotetext{
${ }^{3}$ Incluye las consonantes bilabiales y la labiodental.

${ }^{4}$ Incluye las consonantes dentales y la interdental.

${ }^{5}$ Incluye las oclusivas y la africada.
} 
2. Diptongos. Objeto de estudio: 14 diptongos, 8 crecientes y 6 decrecientes.

\begin{tabular}{|c|c|}
\hline $\begin{array}{c}\text { 1. Intensidad } \\
\text { Átono } \\
\text { Tónico }\end{array}$ & $\begin{array}{c}\text { 2. Estructura silábica } \\
\text { Sílaba abierta } \\
\text { Sílaba cerrada }\end{array}$ \\
\hline $\begin{array}{l}\text { 3. Punto de articulación de la consonante } \\
\text { anterior }\end{array}$ & $\begin{array}{c}\text { 4. Modo de articulación de la consonante } \\
\text { anterior }\end{array}$ \\
Labial & Oclusiva \\
Dental & Fricativa \\
Alveolar & Nasal \\
Velar \\
Palatal & Vibrante \\
Lateral
\end{tabular}

3. Triptongos. Objeto de estudio: 4 triptongos (iai, iei, uai, uei).

\begin{tabular}{|l|c|}
\hline $\begin{array}{l}\text { 1. Punto de articulación de la consonante } \\
\text { anterior }\end{array}$ & $\begin{array}{l}\text { 2. Modo de articulación de la consonante } \\
\text { anterior }\end{array}$ \\
Labial & Oclusiva \\
Dental & Fricativa \\
Alveolar & Nasal \\
Velar & Vibrante \\
Palatal & Lateral \\
\hline $\begin{array}{l}\text { 3. Vibración laríngea de la consonante } \\
\text { anterior }\end{array}$ & \\
Sorda & \\
Sonora & \\
\hline
\end{tabular}

4. Hiatos. Objeto de estudio: 4 tipos distintos de hiatos ${ }^{6}, 21$ posibles combinaciones.

\begin{tabular}{|l|l|}
\hline $\begin{array}{l}\text { 1. Punto de articulación de la consonante } \\
\text { anterior }\end{array}$ & $\begin{array}{l}\text { 2. Modo de articulación de la consonante } \\
\text { anterior }\end{array}$ \\
Labial & Oclusiva \\
Dental & Fricativa \\
Alveolar & Nasal \\
Velar & Vibrante \\
Palatal & Lateral \\
\hline $\begin{array}{l}\text { 3. Vibración laríngea de la consonante } \\
\text { anterior }\end{array}$ \\
Sorda \\
Sonora
\end{tabular}

${ }^{6}$ Vocal abierta átona + vocal cerrada tónica; vocal cerrada tónica + vocal abierta átona; dos vocales iguales; dos vocales abiertas distintas. 
5. Consonantes en posición explosiva. Objeto de estudio: 28 alófonos consonánticos.

\begin{tabular}{|c|r|}
\hline 1. Contexto fónico & 2. Estructura silábica \\
Consonante + vocal & Sílaba abierta \\
Consonante $+[\mathrm{r}]$ & Sílaba cerrada \\
Consonante $+[1]$ & \\
\hline
\end{tabular}

6. Consonantes implosivas $/ l, r, n, s /$. Objeto de estudio: 13 alófonos consonánticos.

\begin{tabular}{|c|c|}
\hline 1. Posición & 2. Contexto fónico \\
Final de sílaba & Delante de vocal \\
Final de palabra & Delante de consonante sorda \\
& Delante de consonante sonora \\
\hline
\end{tabular}

7. Consonantes en posición implosiva. Objeto de estudio: 3 combinaciones de grupos consonánticos ${ }^{7}$ :

Oclusiva sorda+ consonante (/pt/, /ps/, /pn/, /tm/,/tn/, /tl/, /ks/, /kt/)

Oclusiva sonora+ consonante (/bt/, /bs/, /bx/, /bm/,/dx/, /db/, /dm/, /dk/, /gn/)

Nasal + nasal $(/ \mathrm{nn} /, / \mathrm{mn} /, / \mathrm{nm} /)$

8. Acento de intensidad. Objeto de estudio: intensidad fónica.

\begin{tabular}{|c|c|c|}
\hline 1. Intensidad & 2. Lugar del acento & 3. Número de sílabas \\
Tónica & Oxítonas & Monosílaba \\
Átona & Paroxítonas & Bisílaba \\
& Proparoxítonas & Trisílaba \\
& Superproparoxítonas & Tetrasílaba \\
& & Pentasílaba \\
\hline
\end{tabular}

9. Grupos fónicos. Objeto de estudio: formación de grupos fónicos.

\begin{tabular}{|l|c|}
\hline 1. Extensión & 2. Posición \\
Dos palabras & Inicial \\
Tres palabras & Intermedia \\
Cuatro palabras & Final \\
Cinco palabras & \\
Más de cinco palabras & \\
\hline
\end{tabular}

${ }^{7}$ Para el estudio de este aspecto, solo se tendrán en cuenta variables socioculturales.

${ }^{8}$ Por lo tanto, las opciones fueron: (a) monosílaba átona; (b) monosílaba tónica; (c) bisílaba átona; (d) oxítona; (e) paroxítona; (f) proparoxítona; (g) adverbios en -mente. 
10. Entonación. Objeto de estudio: 6 patrones entonativos.

[-interrogativo, -enfático, -suspensión] [+interrogativo, -enfático, -suspensión] [-interrogativo, +enfático, -suspensión] [-interrogativo, -enfático, +suspensión] [+interrogativo, +enfático, -suspensión] [-interrogativo, +enfático, +suspensión]

\begin{tabular}{|c|c|}
\hline $\begin{array}{l}\text { 1. Entorno sintáctico } \\
\text { Oración aislada } \\
\text { Texto } 9\end{array}$ & $\begin{array}{l}\text { 2. Estructura sintáctica } \\
\text { Oración simple afirmativa } \\
\text { Oración simple interrogativa } \\
\text { Oración simple exclamativa } \\
\text { Oración simple con enumeración } \\
\text { Oración con incisos } \\
\text { Oración de relativo } \\
\text { Oración coordinada } \\
\text { Oración yuxtapuesta } \\
\text { Oración principal + subord. } \\
\text { Oración subord. + principal }\end{array}$ \\
\hline
\end{tabular}

\subsubsection{Instrumentos para la recogida de datos (producción y percepción). Los estilos de habla}

El estudio de la adquisición y aprendizaje de una lengua extranjera no puede centrarse exclusivamente en cuestiones de producción, por más que sean éstas fundamentales, sino que debe atender también a la percepción, pues la adquisición fónica de una lengua está condicionada por la forma en que los estudiantes perciben los nuevos sonidos. Para autores como Flege $(1980,1991)$ o Wode $(1994,1996)$, la semejanza o diferencia percibida por el aprendiz entre la L1 y la L2 será determinante en la configuración del nuevo sistema, además de un predictor de las dificultades y de los procesos de transferencia. De manera muy general, se puede señalar que en los casos de sonidos semejantes, el aprendiz asimilará el nuevo elemento a la categoría fonológica de su L1, ante la dificultad de crear una nueva categoría a tan corta distancia fónica. Sin embargo, cuando se trata de un sonido diferente, el aprendiz no lo relacionará con ninguno de su lengua y, por tanto, construirá para él (con mayor o menor acierto) una nueva categoría. Se deduce de esto que un sonido diferente es, comparativamente, más fácil de adquirir que uno semejante.

El corpus AACFELE se compone, sobre todo, de muestras de habla (producción) pero también de datos de percepción. Los instrumentos utilizados para cada caso son, obviamente, de naturaleza muy diferente. Para la recogida de datos de producción nos servimos de grabaciones con distintos grados de formalidad y con diferente nivel atención al habla. De esta forma, se pudo incorporar un nuevo factor de análisis, el del estilo de habla, que permitirá valorar el verdadero alcance

${ }^{9}$ Entiéndase texto como una unidad de comunicación supraoracional con sentido completo e intención pragmática. 
de las dificultades fónicas y la interrelación que existe entre la pronunciación en L2 y la construcción de un discurso oral fluido (hasta qué punto se desatiende la pronunciación cuando el esfuerzo se centra en el mensaje). Para ello, se diseñaron cuatro procedimientos diferentes:

- Breve conversación estructurada en la que dos locutores hablaban sobre un tema que se les proponía, para lo que contaban con la ayuda de una lista de cuestiones sobre las que charlar.

- Lectura de textos; cada locutor grabó dos textos diferentes, uno de carácter dialógico y otro de carácter narrativo.

- Lectura de frases; 36 frases destinadas especialmente al análisis de fenómenos suprasegmentales (grupos fónicos y entonación).

- Lectura de palabras; 297 palabras en las que se recoge la totalidad de las unidades segmentales descritas, en sus diferentes contextos fónicos. También se atiende, en este caso, a cuestiones de intensidad.

Cada locutor participó en dos tipos de sesiones: grabación en parejas (para conversación estructurada) y grabación individual (para la lectura). Se grabó en primer lugar la conversación con objeto de que el resultado fuera el más natural posible. No se explicó a los locutores el objetivo concreto ("estudio de la pronunciación”), sino que se les indicó, simplemente, que era una investigación sobre el aprendizaje del español. Durante la conversación estuvieron más pendientes de cuestiones gramaticales y léxicas que de las fonéticas. En segundo lugar, y de forma individual, se grabó a cada uno de ellos en la lectura, comenzando por los textos para finalizar con las listas de palabras. A medida que avanzaba la sesión se iban percatando de que el objetivo de la investigación era su pronunciación, por lo que se iban esforzando en pronunciar mejor. Con la lectura de palabras se consiguió el máximo nivel de cuidado y atención a los aspectos fónicos. De esta manera, obtuvimos muestras de habla de dos registros ([+ formal] / [- formal]) bien diferenciados.

Con respecto a la recogida de datos de percepción, ésta se llevó a cabo mediante un test de elección múltiple en el que se atendía, de igual manera, a aspectos segmentales que suprasegmentales. Pero a diferencia de los materiales para la producción, de carácter genérico e indiscriminado (salvo en el caso de las palabras, el resto permite el análisis de sonidos, intensidad, grupos fónicos, entonación... en todos los informantes, independientemente de su lengua materna), fue necesario preparar para cada nacionalidad un test diferente compuesto por aquellas cuestiones de especial complejidad. La estructura y duración fue similar en todos los casos:

- 14 preguntas destinadas a la detección de problemas en el vocalismo y el consonantismo; 
- 5 preguntas destinadas a cuestiones de intensidad;

- 4 preguntas centradas en entonación;

- 5 preguntas sobre sus actitudes y valoraciones ante las dos normas lingüísticas presentes en las grabaciones (español centro-peninsular y español caribeño).

Los tests de percepción se realizaron en grandes grupos; se lograron 60 por nacionalidad, lo que proporcionó un total de 360 tests en esta primera fase. Se trató de que todos los parámetros sociales y lingüísticos descritos anteriormente (sexo, edad, nivel de lengua, etc.) estuvieran representados en proporciones similares a las muestras de producción.

\subsection{Las grabaciones}

Las grabaciones se realizaron en formato wav, pues era necesario contar con la mayor calidad sonora posible. Al mismo tiempo, se hizo una copia en formato comprimido mp3, no destinada al análisis acústico.

Con objeto de mejorar los resultados de la grabación (calidad, naturalidad, etc.), la intervención de cada locutor se dividió en nueve partes y se grabó, por tanto, en nueve archivos:

- Conversación

- Texto dialógico

- Texto narrativo

- Sonidos en contacto

- Grupos fónicos

- Entonación

- Vocales

- Consonantes

- Intensidad

En una fase posterior, y con la ayuda de un editor de audio (Audacity 1.3.13 Beta), se realizaron todos los cortes del material, de manera que cada audio correspondiera a una palabra, a una frase o a un texto. A continuación se sometieron a un proceso de limpieza con objeto de eliminar ruidos o ecos, dar realce a las voces, homogeneizar el volumen, etc. En la fonoteca de la página web que alberga el proyecto el corpus aparece fragmentado ya de la manera descrita y depurado.

Para su almacenaje en la base de datos, cada archivo fue identificado con dos códigos: el primero de ellos correspondía a los parámetros utilizados para la definición del corpus y el segundo, al contenido lingüístico. Se acordaron las siguientes convenciones: 


\section{$1^{\circ}$ código sociocultural:}

- Locutor

- L01

- L02

- .

- .

-

- L16

- Nacionalidad

- Pl: Polonia

- Pt: Portugal

- Gr: Grecia

- Cn: China

- De: Alemania

- Eg: Egipto

- Sexo

- $\mathrm{H}$ : hombre

- M: mujer

- Grupo de edad

- 1: grupo 1

- 2: grupo 2

\section{$2^{\circ}$ código lingüístico:}

- Categoría

- conversación

- texto

- frase

- palabra
- Contacto con el español

- a: alto

- m: medio

- b: bajo

- $\quad$ s: muy bajo

- Nivel de lengua

- A2

- B1

- B2

$-\mathrm{C} 1$

- Experiencias de aprendizaje

- si: sí ha trabajado la fonética

- av: algunas veces

- no: nunca o casi nunca

- Aspecto lingüístico (sólo en el caso de las palabras y, parcialmente, en el de las frases) ${ }^{10}$

Palabra

- $\quad$ vocales

- diptongos

- $\quad$ triptongos

- hiatos

- consonantes explosivas

- consonantes implosivas

- grupos consonánticos

- intensidad
Frase

- enunciativa_01/_02/_03...

- interrogativa_01/_02/_03...

- enfática_01/_02/_03...

- suspensión_01/_02/_03...

- Texto grabado (sólo para el caso de las palabras)

${ }^{10}$ Cada palabra se centra en un aspecto concreto de la pronunciación del español. En las frases hay un aspecto principal previsto, relacionado con la entonación o los grupos fónicos, y otros secundarios (sonidos, intensidad) no previstos. Los textos y la conversación proveen de todo tipo de cuestiones, pues ofrecen contextos fónicos más ricos para el análisis. 
Algunos ejemplos del etiquetado final de los archivos son:

L01ChM2aB2si-palabras_vocales_bebo

L12GrM1mB2si-palabras_consonantesimplosivas_cartas

L14PkH1mA1no-frases_interrogativas_09

L08DeH2bC1no-texto_narrativo

\section{CONCLUSIONES}

El componente fónico constituye una pieza más del complejo entramado que es la competencia comunicativa. Cumple un papel fundamental en la actualización de las destrezas orales (expresión, comprensión e interacción) y mantiene relaciones fundamentales con el resto de niveles de la lengua (léxico, sintáctico, textual), de ahí que reivindiquemos su importancia para el aprendizaje de una L2.

Dado el carácter social de la comunicación y teniendo en cuenta que ésta se lleva a cabo, sobre todo, a través de la oralidad, es de destacar el papel que desempeña la correcta pronunciación de una lengua extranjera en las relaciones sociales y en la integración en la comunidad. Es bien conocido lo incómodo que puede resultar hablar con una persona a la que se le entiende mal o que distorsiona constantemente la lengua en su aspecto fónico (lógicamente, el más perceptible). El esfuerzo de comprensión que ha de hacer el interlocutor en estos casos es enorme (nada comparable con el caso de errores gramaticales o léxicos), lo que, sin duda, lleva en muchas ocasiones a un rechazo. Este hecho, que resulta siempre grave, adquiere mayores dimensiones cuando tal rechazo se da en ámbitos profesionales, de servicios públicos, vecindales, educativos.

Por todo ello, la enseñanza de una lengua extranjera, en este caso del español, requiere de una atención a las cuestiones fónicas, al menos similar a la que se presta a otras subcompetencias comunicativas. Sin embargo, lamentablemente, no es esta la práctica habitual, pues son todavía muy escasos los trabajos de investigación que abordan el tema desde esta perspectiva. En realidad, son escasas las investigaciones de carácter aplicado que están orientadas al estudio de la adquisición y del aprendizaje del componente fónico del español como lengua extranjera. Se trata de un área de estudio cuyo vacío es significativo y que urge comenzar a subsanar. Para acometer esta tarea es necesario contar con investigaciones que profundicen en el conocimiento de la competencia fónica, de los procesos de adquisición y aprendizaje que tienen lugar, de la relación entre percepción y producción, de las dificultades fundamentales a las que se deben enfrentar los estudiantes, etc. Al mismo tiempo, se precisan estudios que, partiendo de trabajos como los citados, aborden aspectos de metodología y didáctica de la pronunciación de español 
como lengua extranjera, la elaboración curricular, el desarrollo de materiales, etc. Y previo a todo ello, es, sin duda, contar con corpus de datos orales sobre los que poder investigar y con los que poder desarrollar los trabajos de manera eficaz.

El corpus $A A C F E L E$ es el primero en reunir un número significativo y representativo de grabaciones orales de aprendices de español y el primero en plantearse un estudio general de la pronunciación ELE en correlación con diversos aspectos sociales, culturales y educativos. Ofrece datos no sólo de producción oral, sino también de percepción auditiva. En estos momentos, está en su primera fase (recoge grabaciones de estudiantes de seis nacionalidades), pero es nuestra intención -y nuestro deseo- que vaya ampliándose y, sin duda, mejorándose en los muchos aspectos susceptibles de ello. Confiamos en que pueda resultar un recurso útil para todos los investigadores y profesores que quieran hacer uso de él, y cumpla con los objetivos para los que se ha creado.

\section{REFERENCIAS}

Altman, Howard B. (1980). Foreign language teaching: focus on the learner. En H.B. Altman y C. Vaughan James (eds.), Foreign language teaching: Meeting individual needs (pp. 1-16). Oxford, Inglaterra: Pergamon Press.

Bachman, Lyle. (1995). Habilidad lingüística comunicativa. En M. Llobera (coord.), Competencia comunicativa. Documentos básicos en la enseñanza de lenguas extranjeras (pp. 105-127). Madrid, España: Edelsa.

Canale, Michael y Swain, Merrill. (1980). Theoretical bases of communicative approaches to second language teaching and testing. Applied Linguistics, 1, 1-47. Traducción al español: Fundamentos teóricos de los enfoques comunicativos. Signos, 17, 56-61, y 18, 78-91 (1996).

Cano García, Francisco. (2000). Diferencias de género en estrategias y estilos de aprendizaje. Psicothema, 12(3), 360-367.

Cantero, Francisco José. (1997). De la fonética instrumental a la enseñanza de la pronunciación. En F. J. Cantero, A. Mendoza y C. Romea (eds.), Didáctica de la lengua y la literatura para una sociedad plurilingüe del siglo XXI (pp. 179187). Barcelona, España: Publicacions de la Universitat de Barcelona.

Cantero, Francisco José. (2002). Teoría y análisis de la entonación. Barcelona, España: Publicacions de la Universitat de Barcelona.

Consejo Social Europeo. (2002). Marco común europeo de referencia para las lenguas: aprendizaje, enseñanza, evaluación. Madrid, España: MECD-Instituto Cervantes-Anaya.

Dauer, Rebecca M. (2005). The lingua franca core: A new model for pronunciation instruction? TESOL Quarterly, 39(3), 543-550. 
Derwing, Tracey M.; Rossiter, Marian J.; Munro, Murray J. y Thomson, Ron I. (2004). Second language fluency: Judgments on different tasks. Language Learning, 54, 655-679.

Dieling, Helga y Hirschfeld, Ursula. (2000). Phonetik lehren und lernen. München, Alemania: Langenscheidt Verlag, Fernstudieneinheit.

Elliott, Raymond. (1997). On the teaching and acquisition of pronunciation within a communicative approach. Hispania, 80(1), 95-108.

Ellis, Nick. (1996). Secuencing in SLA. Phonological memory, chunking, and pionts of order. Studies in Second Language Acquisition, 18, 91-126.

Flege, James Emil. (1980). Phonetic aproximation in second language adquisition. Language Learning, 27, 195-216.

Flege, James Emil. (1987). A critical period for learning to pronounce foreign languages? Applied Linguistics, 8, 162-177.

Flege, James Emil. (1991). Perception and production: The relevance of phonetic input to L2 phonologic learning. En T. Huebner y C.A. Ferguson (eds.), Crosscurrents in second language acquisition and linguistic theories (pp. 249-290). Philadelphia, USA: John Benjamins.

García Moutón, Pilar. (2000). Cómo hablan las mujeres. Madrid, España: Arco/ Libros.

Howard, Pierce J. (1999). The owner's manual for the brain: Everyday applications from mind-brain research. Atlanta, USA: Bard Press.

Hu, Fang. (2003). Phonological memory, phonological awareness, and foreign language word learning. Language Learning, 53, 429-462.

Hymes, Dell. (1972). Models of the interaction of language and social life. En J. Gumperz y D. Hymes (eds.), Directions in sociolinguistics: the ethnography of communication (pp. 35-71). New York, USA: Holt, Rinehart, Winston.

Iruela, Agustín. (2004). Adquisición y enseñanza de la pronunciación en lenguas extranjeras. Tesis doctoral. Barcelona, España: Universidad de Barcelona.

Iruela, Agustín. (2007). ¿Qué es la pronunciación? RedELE. Revista on line del Ministerio de Educación, Cultura y Deporte. ISSN: 1571-4667. Disponible en http://www.educacion.gob.es/redele/revista9/articulo_Iruela.pdf.

Johnson, Jacqueline S. y Newport, Elissa L. (1989). Critical period effects in second language learning: The influence of maturational state on the acquisition of English as a second language. Cognitive Psychology, 21, 60-99.

Labov, William. (1996). Principios del cambio lingüistico. Madrid, España: Gredos. Lenneberg, Eric Heinz. (1967). Biological foundations of language. New York: John Wiley and Sons. Traducción española: Fundamentos biológicos del lenguaje. Madrid, España: Alianza Editorial (1975).

Linell, Per. (1982). The concept of phonological form and the activities of speech production and speech perception. Journal of Phonetics, 10, 37-42. 
Lippi-Green, Rosina. (1997). English with an accent: Language ideology and discrimination in the United States. New York, USA: Routledge.

Long, Michael H. (1990). Maturational constraints on language development. Studies in Second Language Acquisition, 12, 251-286.

Moreno, Francisco. (1990). Metodología sociolingüistica. Madrid, España: Gredos. Moreno, Francisco. (2002). Producción, expresión e interacción oral. Madrid, España: Arco/Libros.

Munro, Murray J. (2003). A primer on accent discrimination in the Canadian context. TESL Canada Journal, 20(2), 38-51.

Oyama, Susan. (1976). A sensitive period in the acquisition of a non-native phonological system. Journal of Psycholinguistic Research, 5, 261-285.

Patkowski, Mark S. (1990). Age and accent in second language: A reply to James Emil Flege. Applied Linguistics, 11(1), 73-89.

Poch, Dolors. (2004a). La pronunciación en la enseñanza del Español como Lengua Extranjera. RedELE, Revista on line del Ministerio de Educación, Cultura y Deporte. ISSN: 1571-4667. Disponible en http://www.educacion.gob.es/ redele/revistaRedEle/2004/segunda.html.

Poch, Dolors. (2004b). Los contenidos fonéticos-fonológicos. En J. Sánchez Lobato e I. Santos Gargallo (eds.), Vademecum para la formación de profesores (pp. 753-765). Madrid, España: SGEL.

Scovel, Thomas. (1969). Foreign accents, language acquisition and cerebral dominance. Language Learning, 19, 245-253.

Scovel, Thomas. (1988). A time to speak. A psycholinguistic inquiry into the critical period for human speech. Rowley, MA, USA: Newbury House.

Torres, José Ramón. (2001). Las actividades en los manuales de pronunciación de inglés como lengua extranjera. Biblioteca Phonica, 6. Revista on line de la Universitat de Barcelona.ISSN: 1699-8774. Disponible en www.ub.es/lfa.

Wode, Henning. (1994). Nature, nurture, and age in language acquisition. Studies in Second Language Acquisition, 16, 325-345.

Wode, Henning. (1996). Speech perception and L2 phonological acquisition. En P. Jordaens y J. Lalleman (eds.), Investigating Second Language Acquisition (pp. 321-353). Berlin, Alemania: Mouton de Gruyter. 